\title{
ARTICLE
}

\section{Neutron fluence monitoring system in mono-energetic neutron fields at FRS / JAEA}

\author{
Yoshihiko Tanimura*, Katsutoshi Fujii, Masahiro Tsutsumi and Michio Yoshizawa \\ Nuclear Science Research Institute, Japan Atomic Energy Agency, 2-4 Shirakata, Tokai-mura, Naka-gun, Ibaraki-ken, 319-1195, \\ Japan
}

\begin{abstract}
Mono-energetic neutron calibration fields were developed from $8 \mathrm{keV}$ to $19 \mathrm{MeV}$ using an accelerator at the Facility of Radiation Standards (FRS). Two kinds of neutron detectors are used for monitoring the neutron fluence at a calibration point. One is the Long Counter placed at the 60 degree angle with respect to the beam line except for the 14.8 and $19 \mathrm{MeV}$ fields where the angle is 90 degree. The other is the 110deg-monitor installed in a collimator at 110 degree angle with respect to the beam line. These two monitors were designed to have flat response to the neutrons from a few $\mathrm{keV}$ to a few $\mathrm{MeV}$. The neutron fluence at a calibration point can be evaluated from the counts of the Long Counter with corrections of scattered neutrons. The neutron energy is sensitive to the beam energy especially for the neutrons below a few hundreds of keV. It was found that the beam energy was able to be monitored using the count ratio of two neutron monitors.
\end{abstract}

Keywords: Pelletron accelerator; mono-energetic neutron; calibration field; neutron fluence; Long Counter; neutron monitor; ISO 8529 series

\section{Introduction}

Mono-energetic neutron calibration fields were developed at the Facility of Radiation Standards (FRS) in the Japan Atomic Energy Agency (JAEA) using a 4MV Pelletron accelerator [1-3]. The neutron energies shown in Figure 1 are available for determining energy responses of neutron dosemeters. The neutron energies in these fields correspond to the energy points specified in the international standard (ISO 8529-1) and Japanese industrial standard (JIS Z4521) [4,5].

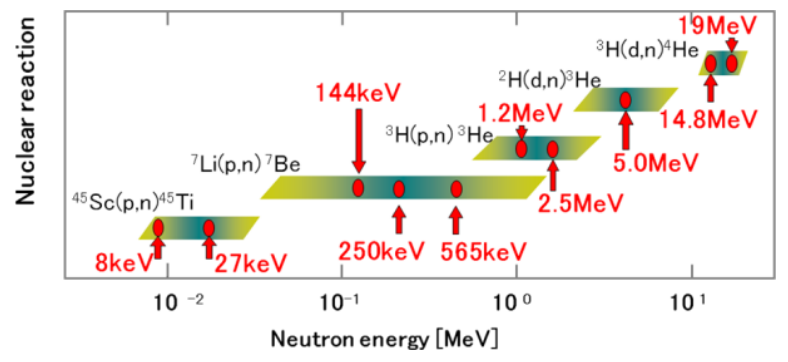

Figure 1. Available energy points and nuclear reactions employed for neutron production in the mono-energetic neutron calibration fields.

Precise neutron fluence is the most important parameter for calibration of dosemeters. Since emission rate of accelerator-produced neutron would vary with time, neutron emission rates must be monitored during irradiation. Then the irradiated neutron fluence should be determined from a neutron monitor placed in the field.

We developed a neutron monitoring system and installed it in our fields. This paper describes the system and the procedure to determine the neutron fluence.

\section{Arrangement in neutron fields}

Figure 2 shows an arrangement in the mono-energetic neutron fields. Dosemeters are placed at a calibration point which is set at an angle of 0 degree with respect to the beam line except for the $14.8 \mathrm{MeV}$ field, where the angle is 45 degree. In order to determine neutron fluence at the calibration point a Long Counter (hereinafter LC) was installed in the field for monitoring neutron emission rate from the target. The LC is used as a primary monitor for the neutron fluence measurement and is placed at $220 \mathrm{~cm}$ from the target at the 60 degree angle with respect to the beam line except for the 14.8 and $19 \mathrm{MeV}$ fields. For these two energies the LC is placed at $300 \mathrm{~cm}$ from the target at 90 degree angle. Another neutron detector with flat response (hereinafter 110deg-Monitor) has been installed as a secondary monitor in a collimator on the 110 degree angle with respect to the beam line. The distance from the target to the $110 \mathrm{deg}$-monitor is $480 \mathrm{~cm}$.

*Corresponding author. Email: tanimura.yoshihiko@jaea.go.jp 


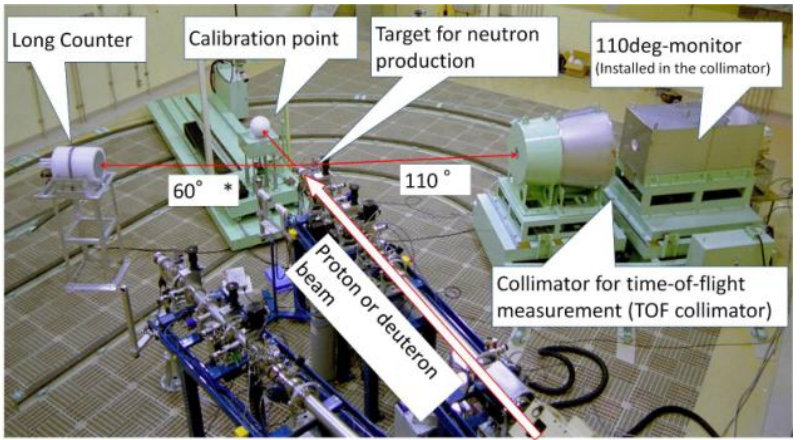

Figure 2. Photograph in the mono-energetic neutron fields. *In the 14.8 and $19 \mathrm{MeV}$ fields, the Long Counter is placed at 90 degree angle to the beam line.

\section{Characteristics of neutron monitors}

\subsection{Long Counter}

Figure 3 shows a schematic drawing of the Long Counter. It consists of a thermal neutron detector and a cylindrical moderator made of polyethylene with a diameter of $21.8 \mathrm{~cm}$ and a length of $31.8 \mathrm{~cm}$. The moderator is surrounded with a $0.5 \mathrm{~mm}$ thick $\mathrm{Cd}$ sheet and an outer shield made of polyethylene with a thickness of about $8 \mathrm{~cm}$ except for the entrance face of the neutron produced in the target.

$\mathrm{A} \mathrm{BF}_{3}$ proportional counter is used as the detector except for the 8 and $27 \mathrm{keV}$ fields. It has a sensitive region with $25.4 \mathrm{~mm}$ in diameter and $318 \mathrm{~mm}$ in length. As the neutron yield is low in the 8 and $27 \mathrm{keV}$ fields, a ${ }^{3} \mathrm{He}$ proportional counter, which has higher sensitivity than the $\mathrm{BF}_{3}$ counter, is employed for these energies in order to increase the detection efficiency of the LC.

There are six polyethylene rods to introduce additional holes in the front surface of the polyethylene moderator. These holes allow lower-energy neutrons to penetrate farther into the moderator and prevent the neutrons from escaping from the front surface. This increases the efficiency for the lower-energy neutrons [6]. Figure 4 shows the response function of the LC calculated using the MCNP-4C code [7]. The depths of the holes were determined to be $20 \mathrm{~cm}$ which makes the flattest response in the calculations with different depth. From the Figure 4, the response of the LC was found to be constant within $10 \%$ in the neutron energy from a few $\mathrm{eV}$ to a few MeV.

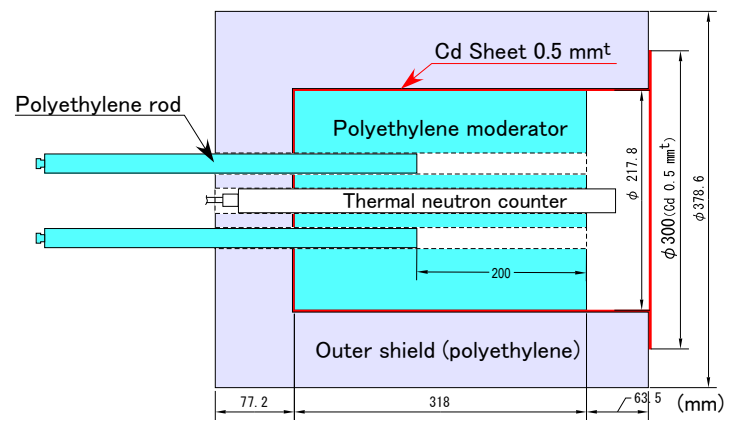

Figure 3. Schematic drawing of the Long Counter.

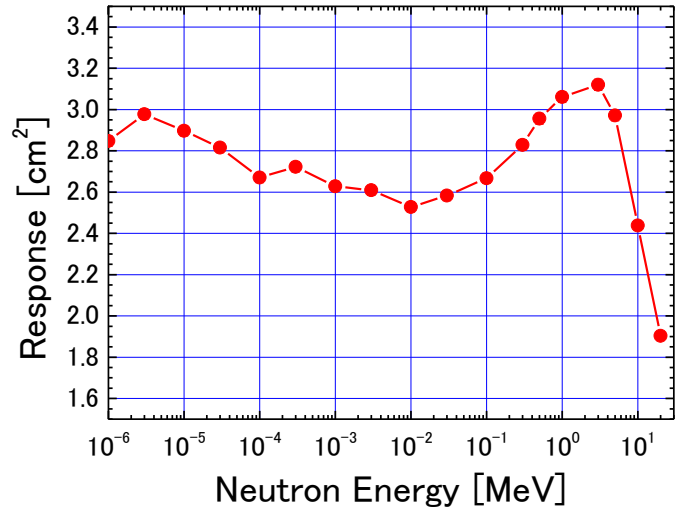

Figure 4. Response of the Long Counter calculated using the MCNP-4C code. The response was determined from the number of ${ }^{10} \mathrm{~B}(\mathrm{n}, \alpha)^{7} \mathrm{Li}$ reaction in the sensitive area of the $\mathrm{BF}_{3}$ counter. The shape of the response curve for the ${ }^{3} \mathrm{He}$ counter is quite similar to that of the $\mathrm{BF}_{3}$ counter.

\subsection{0deg-monitor}

The 110deg-monitor has been installed in a collimator for time-of-flight measurement (TOF collimator) as shown in Figure 5. It consists of a ${ }^{3} \mathrm{He}$ proportional counter and a cylindrical moderator made of polycarbonate and polyethylene. The counter has a sensitive region with $25.4 \mathrm{~mm}$ in diameter and $305 \mathrm{~mm}$ in length, and the pressure of the ${ }^{3} \mathrm{He}$ gas is $0.5 \mathrm{MPa}$. The dimensions of the polycarbonate moderator are 22 $\mathrm{cm}$ in diameter and $10 \mathrm{~cm}$ in length. Those of the polyethylene moderator are $22 \mathrm{~cm}$ in diameter and 20.5 $\mathrm{cm}$ in length. Neutrons produced in the target are narrowed to $14 \mathrm{~cm}$ in diameter by the TOF collimator and enter into a surface of the polycarbonate moderator. As the hydrogen density of polycarbonate is approximately half that of polyethylene, lower-energy neutrons can penetrate the moderator farther than that made of polyethylene. This increases the detection efficiency for the lower energy neutrons [8]. Figure 6 shows the response function of the 110deg-monitor calculated using the MCNP-4C code. The response function was found to be flat enough to be used as a neutron monitor in the neutron energy range from a few $\mathrm{keV}$ to a few $\mathrm{MeV}$, where this monitor was mainly used.

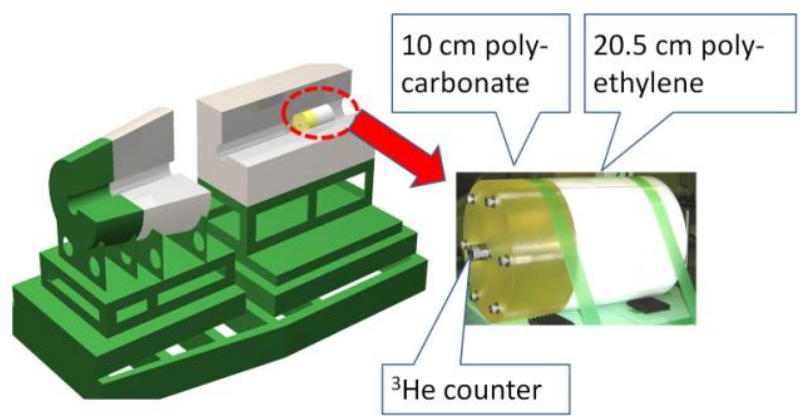

Figure 5. Schematic drawing and photograph of the $110 \mathrm{deg}$-monitor installed in the collimator for time-of-flight measurement. 


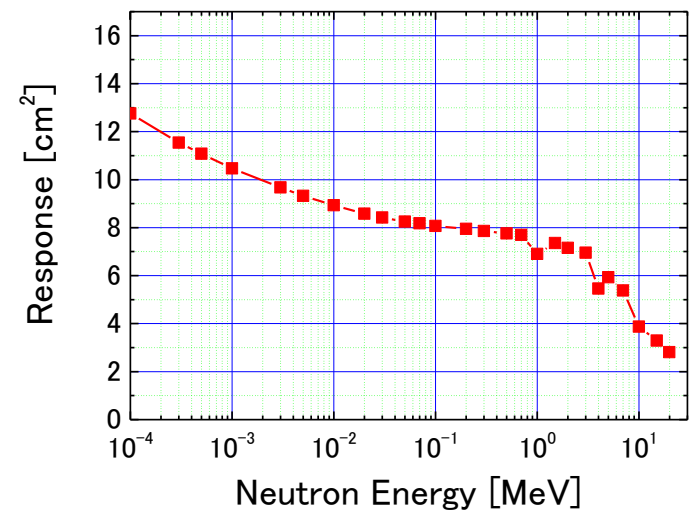

Figure 6. Response of the 110deg-monitor calculated by using the MCNP-4C code. The response was determined from the number of ${ }^{3} \mathrm{He}(\mathrm{n}, \mathrm{p})^{3} \mathrm{H}$ reaction in the sensitive area of the ${ }^{3} \mathrm{He}$ counter.

\section{Neutron fluence monitoring system}

Figure 7 shows a block diagram of a fluence monitoring system developed in this work. It was designed to record the each counts of the LC and the $110 \mathrm{deg}$-monitor every $10 \mathrm{~s}$. In addition, beam current, room temperature, humidity and atmospheric pressure are also logged by this system. These data are important to check the stability of the neutron fluence rate during calibration of dosemeters.

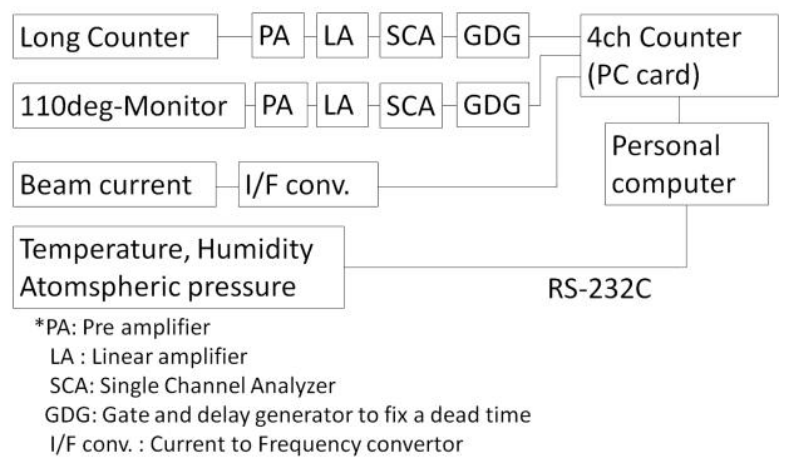

Figure 7. Block diagram of the developed neutron fluence monitoring system.

\section{Determination of neutron fluence}

The neutron fluence $\phi(d)$ at the calibration point, which is $d \mathrm{~cm}$ from the target, can be derived by the following equation:

$$
\phi(d)=\frac{N \times K \times S \times F_{A}(d)}{d^{2}}
$$

where $N$ is the counts of the neutron monitor; $K$ is the calibration coefficient from the $N$ to neutron fluence per steradian; $S$ is the factor for correcting the contribution of incident neutrons into the monitor by scattering from instruments to be calibrated and their supporting materials; $F_{A}(d)$ is the correction factor for air attenuation of neutrons. The calibration coefficients $K$ were determined for every energy point by measuring the reference neutron fluence at the calibration point using neutron detectors such as a Bonner sphere, a proton recoil proportional counter and a semiconductor detector with a polyethylene convertor [1-3]. The correction factor $S$ is determined for every calibration by measuring the ratio of monitor counts with and without the instruments and their supporting materials.

The detection efficiency of the $110 \mathrm{deg}$-monitor is too low to obtain sufficient count during the calibration due to the long distance from the target. Therefore, we decided to use the LC as a primary monitor to determine the neutron fluence.

The correction factor $F_{A}(d)$ was calculated using the MCNP-ANT code [9], which simulated the transport of charged particles and the neutron production in the target as well as the neutron transport. Figure 8 shows the relationships between the $F_{A}(d)$ and the distance $d$ from the target. It can be found from the figure that the $F_{A}(d)$ is almost proportional to the $d$ for all the energies. Then the functions of the $F_{A}(d)$ was determined for each energy from the regression line to fit the calculated data shown in Figure 8.

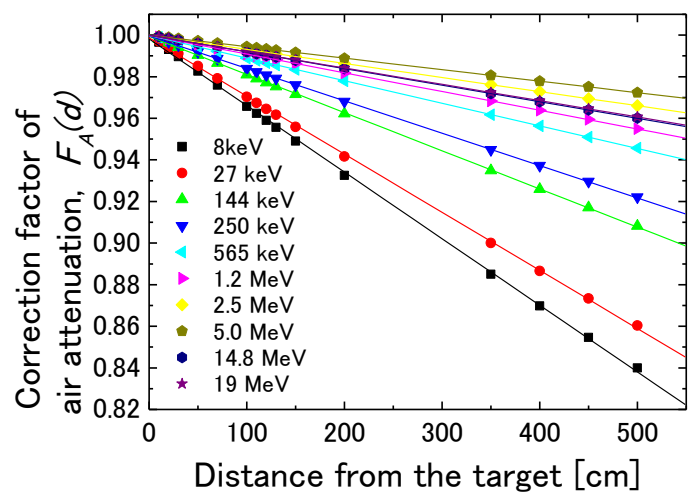

Figure 8. Correction factor of the air attenuation $F_{A}(d)$ as a function of the distance from the target. These data were calculated using the MCNP-ANT code.

\section{Energy monitoring}

If the energy of the proton or deuteron beam changed, the neutron energy would change corresponding to the beam energy. This causes large uncertainty of neutron energy especially in the neutron fields below a few hundreds of $\mathrm{keV}$. Therefore, the neutron energy should be checked in such fields. However, it is not realistic to estimate the neutron energy by time-of-flight measurements at every calibration because such measurement needs much time.

For the 8 and $27 \mathrm{keV}$ fields, the aimed neutron can be produced by adjusting the proton energy to the resonance peak of the ${ }^{45} \mathrm{Sc}(\mathrm{p}, \mathrm{n}){ }^{45} \mathrm{Ti}$ reaction with a target 
voltage control system [2].

For the 144, 250 and $565 \mathrm{keV}$ fields, the count ratio of the two neutron monitors located on different angles can be used to estimate the neutron energy because angular distribution of the ${ }^{7} \mathrm{Li}(\mathrm{p}, \mathrm{n})^{7} \mathrm{Be}$ cross section depends on the proton energy $[10,11]$. Figure 9 shows the count ratios of the LC to the 110deg-monitor as a function of proton energy. These data were obtained by changing the incident proton energy with the target voltage control system. It was found that the proton energy was able to be estimated by measuring the ratio of the monitors. The solid line in Figure 9 represents the ratio of double differential cross sections for the ${ }^{7} \mathrm{Li}(\mathrm{p}, \mathrm{n}){ }^{7} \mathrm{Be}$ reaction between 60 and 110 degree [11]. The energy dependence of the monitor ratio is quite similar to that of the cross section. This means that this characteristics can be explained by the cross section of the ${ }^{7} \mathrm{Li}(\mathrm{p}, \mathrm{n})^{7} \mathrm{Be}$ reaction.

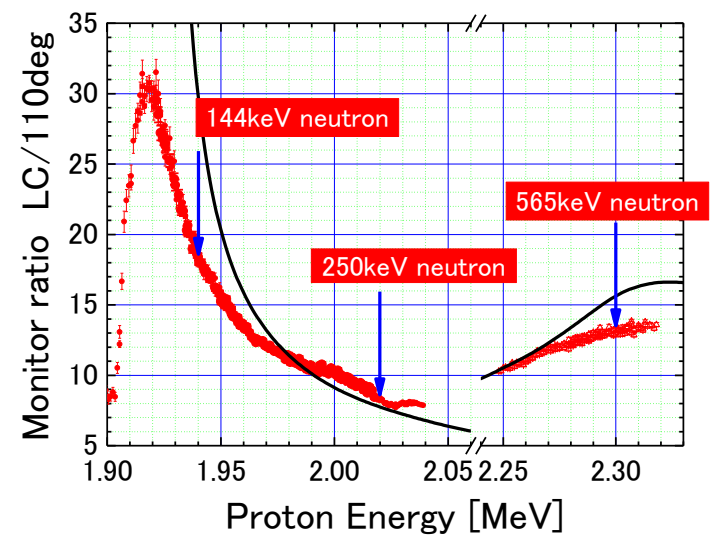

Figure 9. Relationship between proton energy and count ratio of the $\mathrm{LC}$ and the $110 \mathrm{deg}$-monitor for ${ }^{7} \mathrm{Li}(\mathrm{p}, \mathrm{n}){ }^{7} \mathrm{Be}$ reaction.

\section{Summary}

Two neutron monitors were installed in the mono-energetic neutron calibration fields at FRS / JAEA. One is the Long Counter which is used to determine the neutron fluence at calibration point. The other is the $110 \mathrm{deg}$-monitor which has been installed in the TOF collimator as a secondary monitor. These two monitors are designed to have almost flat response to the neutrons from a few $\mathrm{keV}$ to a few $\mathrm{MeV}$. The counts of these monitors are recorded with the developed monitoring system every $10 \mathrm{~s}$. The neutron fluence at the calibration point can be determined from the counts of the LC with corrections of scattered neutrons and air attenuation. The neutron energy is sensitive to the proton energy especially for the energy region below a few hundreds of $\mathrm{keV}$. It was found that the count ratio of the two neutron monitors was able to be used to estimate the proton energy. The developed monitoring system can provide reliable neutron fluence at a calibration point.

\section{Acknowledgements}

The authors would like to thank Drs. M. Yoshida and Y. Yamaguchi for their helpful advice. The authors wish to express their appreciation to Messrs. S. Shimizu, K. Kawasaki, K. Umino, Y. Tatebe and Dr. M. Kowatari for operating and maintain the accelerator and for their assistance during the irradiation.

\section{References}

[1] Y. Tanimura, M. Yoshizawa, J. Saegusa, K. Fujii, S. Shimizu, M. Yoshida, Y. Shibata, A. Uritani and K. Kudo, Construction of $144,565 \mathrm{KeV}$ and 5.0 MeV Monoenergetic Neutron Calibration Fields at JAERI, Radiat. Prot. Dosim., 110 (2004), pp. 85-89.

[2] Y. Tanimura, J. Saegusa, Y. Shikaze, M. Tsutsumi, S. Shimizu and M. Yoshizawa, Construction of Monoenergetic Neutron Calibration Fields Using ${ }^{45} \mathrm{Sc}(\mathrm{p}, \mathrm{n}){ }^{45} \mathrm{Ti}$ Reaction at JAEA, Radiat. Prot. Dosim., 126 (2007), pp. 8-12.

[3] Y. Shikaze, Y. Tanimura, J. Saegusa, M. Tsutsumi, S. Shimizu, M. Yoshizawa and Y. Yamaguchi, Development of the Neutron Calibration Fields using Accelerators at FRS and TIARA of JAEA, $J$. Nucl. Sci. Technol., Suppl. 5 (2008), pp. 209-212.

[4] ISO, Reference neutron radiations - Part 1: Characteristics and methods of production, ISO 8529-1, (2001).

[5] Japanese Industrial Standard, Method of calibration for neutron dose equivalent (rate) meters, JIS Z 4521 : 2006 (2006).

[6] A. O. Hanson and J. L. McKibben, A neutron detector having uniform sensitivity from $10 \mathrm{keV}$ to $3 \mathrm{MeV}$, Phys. Rev., 72 (1947), pp. 673-677.

[7] J. F. Briesmeister (Ed), MCNP - a general Monte Carlo n-particle transport code version $4 C$, LA-13709-M (2000).

[8] Y. Tanimura, J. Saegusa, M. Yoshizawa and M. Yoshida, Design of a single moderator-type neutron spectrometer with enhanced energy resolution in the energy range from a few to 100keV, Nucl. Instr. Meth. Phys. Res. A, A547 (2005), pp. 592-600.

[9] M. Yoshizawa, T. Sugita, J. Saegusa, Y. Tanimura and M. Yoshida, A Monte Carlo program for estimating characteristics of neutron calibration fields using a pelletron accelerator, J. Nucl. Sci. Technol., Suppl. 2 (2002), pp. 1240-1243.

[10]K. Kudo, A. Fukuda, Y. Kawada, N. Kobayashi and N. Hayashi, Facilities for neutron standardization at the Electrotechnical Laboratory, Bulletin of the Electrotechnical Laboratory, 54 (1990), pp. 248-262.

[11]M. Drosg, DROSG-2000: Neutron source reactions, IAEA-NDS-87 (2005). 